\title{
Low-cost and compact single-photon counter based on a CMOS SPAD smart pixel
}

\author{
Gianluca Boso, Member, IEEE, Mauro Buttafava, Student Member, IEEE, Federica Villa, \\ Member, IEEE, and Alberto Tosi, Member, IEEE
}

\begin{abstract}
We present a single-photon counter based on a silicon Single-Photon Avalanche Diode (SPAD) fabricated in a $0.35 \mu \mathrm{m}$ CMOS technology. The detector is monolithically integrated with a front-end circuit and a digital pulse output driver. External components are kept to a minimum and the resulting instrument is low-cost, low-power and compact, being housed into an industry-standard 1-inch aluminum optical mounting tube. It features a maximum power consumption of just $250 \mathrm{~mW}$ from an USB link. The embedded $50 \mu \mathrm{m}$ diameter SPAD has high photon detection efficiency in the visible range $(55 \%$ at $420 \mathrm{~nm})$, low noise $(<\mathbf{1 0 0} \mathrm{cps}$ at room temperature), low timing jitter $(<100 \mathrm{ps}$ full-width at half maximum), and very low afterpulsing probability (down to $1 \%$ with $60 \mathrm{~ns}$ hold-off time). The high performance, compactness and low cost enable many unexplored applications in life sciences, personal health care, industrial quality check, quantum physics and others, where it is required to count single photons and to measure their arrival time.
\end{abstract}

Index Terms - photodetector, single-photon avalanche diode, SPAD, photon counting, near-infrared detector.

\section{INTRODUCTION}

$\mathrm{R}^{\mathrm{c}}$ ecently, there is an increasing number of applications that are based on the acquisition of light signals in the visible and near-infrared range with single-photon sensitivity and sharp (few tens of picoseconds) timing resolution. In fact, light is a powerful tool to probe different properties of a sample, like in many clinical applications [1],[2], fluorescence microscopy [3], quality assessment of food, wood and pharmaceuticals [4],[5]. Moreover, light is used to measure speed and distance of an object, like in 3D ranging for automotive vision [6].

Many different detectors have been reported in literature, featuring single-photon sensitivity and high timing resolution [7]: photomultiplier tubes (PMT), hybrid detectors, semiconductor-based devices like single-photon avalanche diodes (SPAD) or silicon photomultipliers (SiPM), until recent

Manuscript received April 24, 2015.

Gianluca Boso was with the Dipartimento di Elettronica, Informazione e Bioingegneria, Politecnico di Milano, Milano 20133, Italy. He is now with the Group of Applied Physics, University of Geneva, Geneva CH-1211, Switzerland (e-mail: gianluca.boso@polimi.it).

Mauro Buttafava, Federica Villa, and Alberto Tosi are with the Dipartimento di Elettronica, Informazione e Bioingegneria, Politecnico di Milano, Milano 20133, Italy (e-mail: mauro.buttafava@polimi.it; federica.villa@polimi.it; alberto.tosi@polimi.it) developments on cryogenic detectors like superconducting single-photon detectors (SSPD). Depending on the specific technology, it is possible to achieve photon detection efficiency up to $90 \%$ at selected wavelengths within the visible and near-infrared range $(400 \mathrm{~nm}-1700 \mathrm{~nm})$, with low noise (few counts per second) and high timing resolution (few tens of picoseconds).

However, most of the detectors presented in literature have been developed for research and laboratory use, favoring performance over dimension, cost and scalability. This strongly limits the diffusion of single-photon detectors in fastgrowing and large-scale applications, like personal health monitoring, life science, industrial in-line quality check, spectroscopy, quantum computing, automotive vision, etc. Here we present a robust single-photon counter instrument based on a CMOS pixel with SPAD detector and in-pixel electronics, in a compact, low-power and low-cost module, suitable for widespread exploitation.

\section{PIXEL ARCHITECTURE}

The strength of our module consists in the integration of a mixed passive-active quenching circuit together with a SPAD detector in the same chip, fabricated in a cost-effective $0.35 \mu \mathrm{m}$ HV CMOS technology (Fraunhofer IMS, Germany). Besides the main advantages of compact dimension and cost reduction, an integrated quenching circuit brings other important improvements to SPAD performance: the lag in avalanche sensing is reduced and the current flowing through the detector is quickly quenched, thus lowering the afterpulsing probability [8]. Fig. 1 shows a simplified diagram of the front-end circuit: it relies on the progressive quenching of the avalanche current through a time-varying Variable-Load Quenching Circuit (VLQC) [9]: only three high-voltage MOS transistors are connected to the SPAD anode, thus minimizing the overall parasitic capacitance at that node.

Transistor $\mathrm{M}_{\mathrm{S}}$ is normally kept with a quiescence resistance of few tens of $\mathrm{k} \Omega$. When an avalanche is triggered, the SPAD current is quenched through $\mathrm{M}_{\mathrm{S}}$ as the current builds up. The rising edge of the anode voltage is sensed by transistor $\mathrm{M}_{\mathrm{T}}$, which promptly lowers the gate of transistor $\mathrm{M}_{\mathrm{S}}$, thus completely quenching the avalanche current. At the same time, the integrated circuit provides a digital pulse to the OUT pin for counting (i.e. providing intensity measurements) or timing (i.e. measuring the photon arrival time) single photons. 


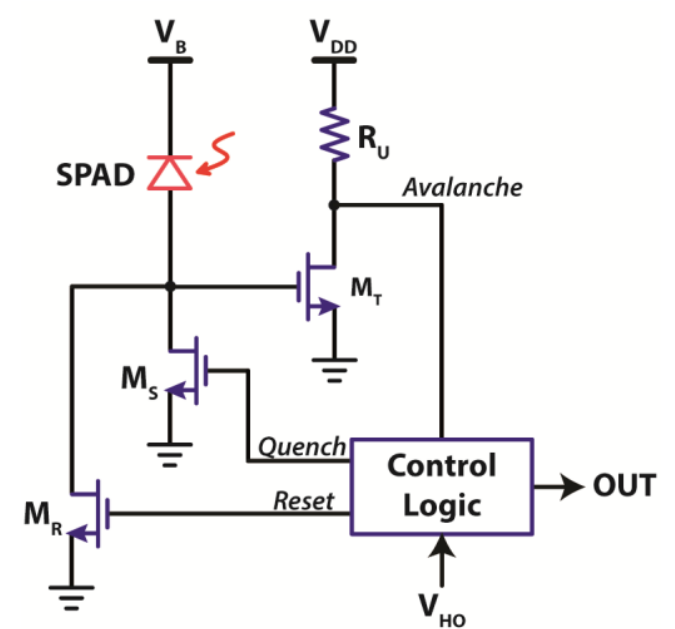

Fig. 1. Smart pixel based on a VLQC architecture with an integrated SPAD.

After each avalanche, the SPAD is kept OFF for few tens of nanoseconds (hold-OFF phase) in order to reduce the afterpulsing effect, then a reset pulse is applied to the gate of transistor $\mathrm{M}_{R}$, which is sized in order to rapidly discharge the parasitic capacitance at the anode. After the reset phase, the initial conditions of the circuit are restored and the SPAD is ready for another detection. The hold-OFF time is adjustable down to a minimum of $20 \mathrm{~ns}$, corresponding to a saturated counting rate of $50 \mathrm{Mcps}$. The avalanche is sensed in few hundreds of picoseconds, then quenched in about $1 \mathrm{~ns}$ and the SPAD is reset in less than $3 \mathrm{~ns}$.

\section{INSTRUMENT DESCRIPTION}

Fig. 2 shows the block diagram of the proposed singlephoton counter. Given the high level of integration of the chip, only a limited number of discrete components is necessary. A standard micro-USB connection is used as both power supply and data link. An 8-bit microcontroller manages the USB protocol. A bias voltage generator, consisting of two lowpower DC/DC converters, provides the $3.3 \mathrm{~V}$ rail to operate the logic gates inside the chip and $\mathrm{V}_{\text {BIAS }} \sim 30 \mathrm{~V}$ to reverse bias the SPAD with an excess voltage (i.e. voltage above breakdown) of $6 \mathrm{~V}$.

The chip outputs a digital pulse, synchronous with the avalanche triggering, routed to the microcontroller and to the high-speed output buffer. Inside the microcontroller, a counter measures the incoming photon flux (i.e. count rate) with a programmable integration time from $10 \mathrm{~ms}$ up to $60 \mathrm{~s}$. The number of counts is streamed to a PC via USB.

A high-speed buffer provides the photon OUT signal and drives a $50 \Omega$ coaxial cable with either NIM (Nuclear Instrumentation Module) or LVTTL (Low-Voltage TransistorTransistor Logic) levels, which are user-selectable through a jumper switch. We exploited a Current-Mode Logic (CML) comparator with NIM-compatible outputs (matching the typical input of time-correlated single-photon counting (TCSPC) systems [10]) and a high-speed LVTTL buffer.

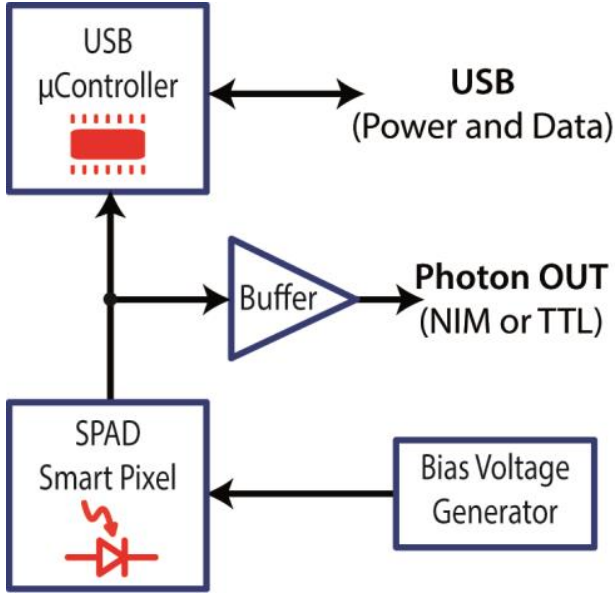

Fig. 2. Block diagram of the single-photon counter module.

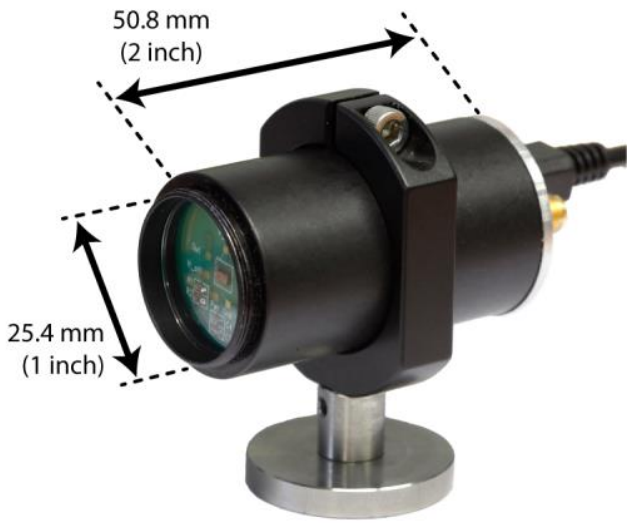

Fig. 3. Overall module, housed in a 1-inch standard aluminum tube and hold by a commercial optics mounting.

Fig. 3 shows the actual working prototype of the detection module. Compared with other commercial silicon singlephoton detectors [11]-[13], it has the smallest form factor since it is housed in a 1-inch diameter aluminum tube with 2-inch length. The chip is directly wire-bonded on a printed circuit board (PCB) and a glass window protects the SPAD active area. The remaining components are placed on a second horizontal PCB, which also features the USB connector for power and data transfer, and the SMA connector for the photon OUT signal.

Table 1. Measured dark count rate, timing jitter, photon detection efficiency, and hold-off time for $1 \%$ afterpulsing probability for different active area diameters of the SPAD integrated in the CMOS smart pixel. All measurements are at room temperature $\left(25^{\circ} \mathrm{C}\right)$ and $6 \mathrm{~V}$ excess bias [14].

\begin{tabular}{|c|c|c|c|c|}
\hline \multicolumn{2}{|c|}{ Active Area Diameter $(\mu \mathrm{m})$} & 50 & 100 & 200 \\
\hline \multicolumn{2}{|c|}{$\mathrm{DCR}(\mathrm{cps})$} & 100 & 320 & 6500 \\
\hline \multicolumn{2}{|c|}{ Timing jitter FWHM (ps) } & 99 & 295 & 610 \\
\hline \multirow{3}{*}{$\operatorname{PDE}(\%)$} & $300 \mathrm{~nm}$ & & 20 & \\
\hline & $420 \mathrm{~nm}$ & & 55 & \\
\hline & $850 \mathrm{~nm}$ & & 5 & \\
\hline \multicolumn{2}{|c|}{$\begin{array}{l}\text { Hold-off time (ns) } \\
\text { for afterpulsing < } 1 \%\end{array}$} & 60 & 100 & 150 \\
\hline
\end{tabular}




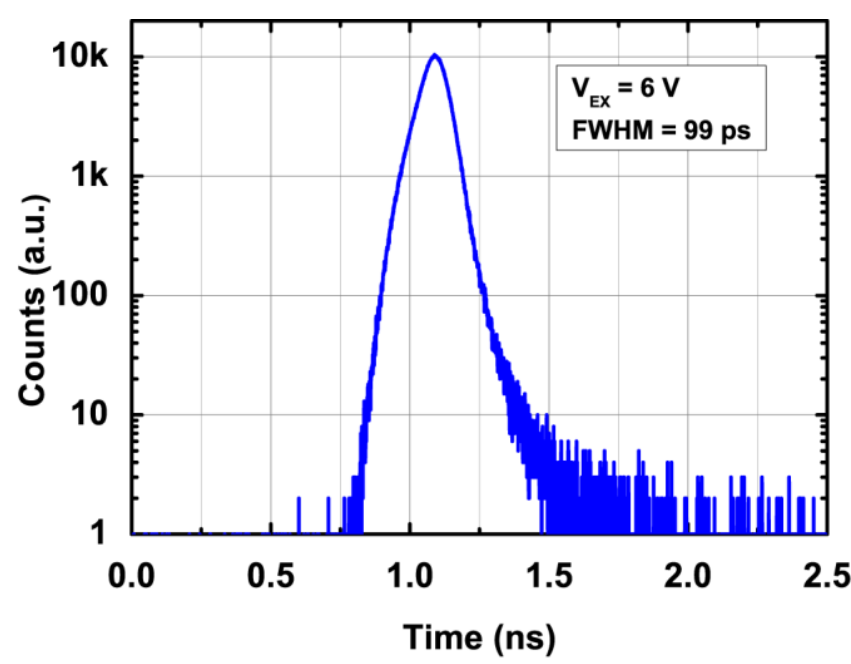

Fig. 4. Time response of the $50 \mu \mathrm{m}$ detector to a $20 \mathrm{ps}$ (FWHM) pulsed laser at $850 \mathrm{~nm}$ with a repetition frequency of $1 \mathrm{MHz}$.

Being housed in a standard form-factor case, the instrument can be easily coupled with different optical accessories, simplifying its assembly in existing experimental setups, for a wide variety of on-field applications.

\section{EXPERIMENTAL CHARACTERIZATION}

The module can house chips with different SPAD active area diameters. Table 1 summarizes the performance of available pixels with diameter of $50 \mu \mathrm{m}, 100 \mu \mathrm{m}$ and $200 \mu \mathrm{m}$ [14], in terms of Dark Count Rate (DCR), timing jitter, Photon Detection Efficiency (PDE), and afterpulsing probability. A more complete characterization of the devices can be found in Ref. [14]. The pixel with a $50 \mu \mathrm{m}$ SPAD was chosen as the best compromise between PDE, DCR and timing resolution, and it is the device tested in the following measurements (its breakdown voltage is $26.5 \mathrm{~V}$ and it was operated at an excess bias voltage of $\mathrm{V}_{\mathrm{EX}}=6 \mathrm{~V}$ ).

\section{A. Timing resolution}

The chip outputs a LVCMOS pulse, synchronous with the avalanche pulse, which is converted by the module to a NIM output signal that can be acquired by any commercial TCSPC system in order to reconstruct the waveform of the optical signal from the histogram of the photon arrival time [10].

Fig. 4 shows the time histogram acquired by a commercial TCSPC board (SPC-130, Becker-Hickl GmbH, Germany) with our detector illuminated by a 20 ps (FWHM) pulsed laser source at $850 \mathrm{~nm}$ with $1 \mathrm{MHz}$ repetition frequency. The overall response of the system is below $100 \mathrm{ps}$ and is dominated by the timing uncertainty of the detector under test. This result is comparable with the value measured with the same SPAD chip (including both the SPAD and the front-end circuit) in other bulky experimental setups [14].

\section{B. Linearity}

Fig. 6 shows the relationship between incoming photon flux and count rate measured with the SPAD module: with a hold-OFF time of $100 \mathrm{~ns}$, the detector saturates at $~ 10 \mathrm{Mcps}$, resulting in a dynamic range (i.e. ratio between maximum count rate and dark count rate) of more than 5 decades. The module is linear up to $\sim 1$ Mcps. The slight deviation in linearity at low count rates is a measurement artifact due to the calibration of the optical setup (power meter, attenuators, lenses) at low photon fluxes.

\section{Stability}

Another important parameter for wide-scale applications is the stability of the count rate over time. Any fluctuation in the supply voltage or in the read-out electronics (e.g. due to temperature drifts) can directly affect the measured photon number, thus possibly impairing the acquisition. Fig. 6 shows the measured photon rate when the SPAD is illuminated with a continuous wave light source, over a period of 2 hours. The normalized peak-to-peak variation in the measured photon count is better than $0.22 \%$, making the detection module suitable even for very demanding applications (e.g. clinical ones), where system stability must be better than $0.5-1 \%$ [2]. The long-term stability ( $>1000$ hours) is expected to be similar under standard laboratory conditions.

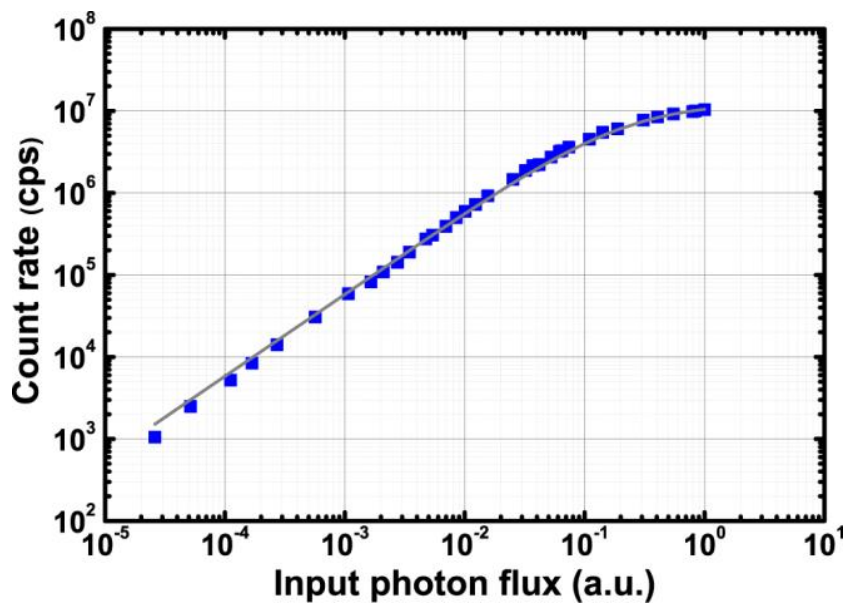

Fig. 5 Dependence of the SPAD count rate on the photon flux (blue diamonds). With a hold-OFF time of $100 \mathrm{~ns}$, the module saturates at $10 \mathrm{Mcps}$. The measured response matches the theoretical trend (grey line).

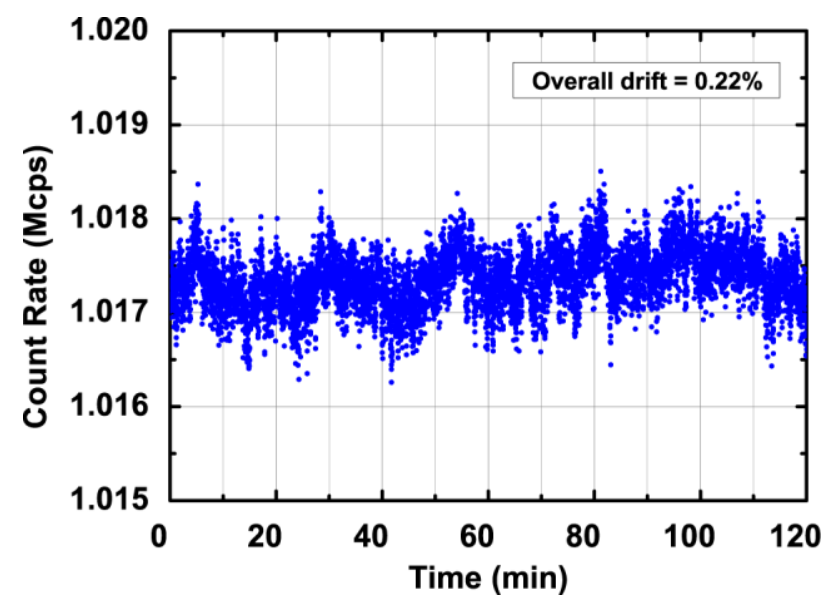

Fig. 6. Measured count rate over a period of 120 minutes with the detector illuminated by a constant $\mathrm{CW}$ light source. The overall variation in the count rate is $<0.22 \%$ over the two hours. 


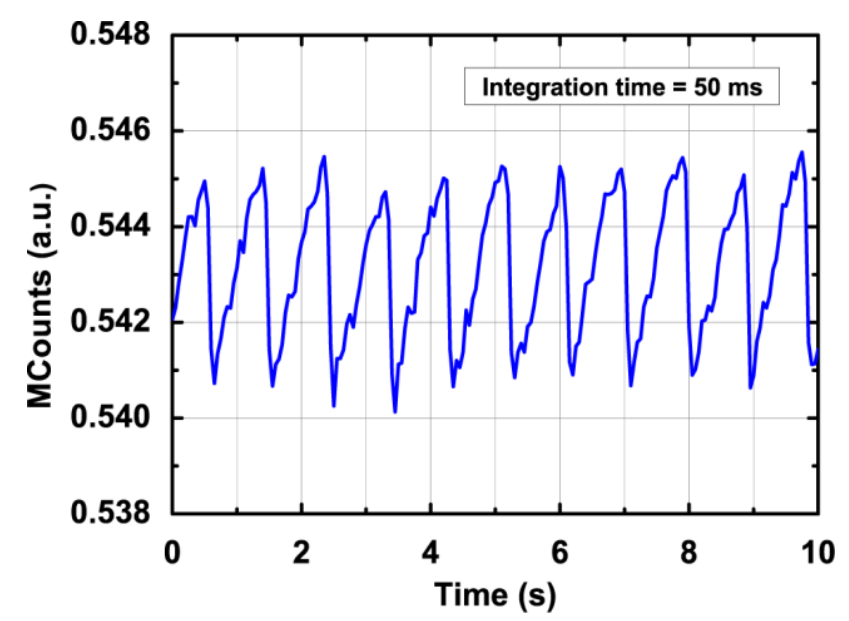

Fig. 7. Count rate plot, as measured by the SPAD detection module during heart-beat measurements. The periodic variation (in the order of $0.75 \%$ of the mean value) represents the changes in the optical properties of the finger (i.e. the blood volume in the vessels) due to the heart-beat (65 bpm in this case).

\section{Application example: heart-rate monitor}

In order to assess the performance of the module in a real-life application, we employed it in a simple setup to measure the variation in time of the total blood absorption, hence the heart rate of a healthy volunteer. This application is a concrete example where a personal health care application could benefit from the use of a single-photon detector. In fact, compared to analog photodetectors, our SPAD pixel has an enhanced sensitivity and dynamic range (hence it can employ a faint, low-power light source) and directly outputs a digital signal, easily processed by a microcontroller. Compared to a standard analog front-end, usually employing a linear-mode photodiode (with a variable-gain trans-impedance amplifier, an analog-to-digital converter, and digital-to-analog converter for offset compensation and calibration), this is a definitive advantage in terms of reliability, reproducibility and cost.

A commercial smartphone flash LED was used to illuminate the index finger of a healthy volunteer and the optical glass window of the detector was placed on the same finger but on the opposite side (transmittance topology). The count rate over 200 consecutive $50 \mathrm{~ms}$ time intervals was acquired. All data were collected by a PC through the USB link and a LabView interface. Fig. 7 shows the acquired curve, where the variation in the absorbance due to a change in the total blood volume in the finger vessels is clearly visible and has a periodicity of approximately 65 beats per minute (bpm), i.e. the heartbeat of the patient. Even if the measured variation in the count rate is just $0.75 \%$ over the average signal (the patient was in standard open-air illuminated environment), pulses are clearly visible, thus proving its high dynamic range.

\section{CONCLUSION}

We designed and characterized a low cost and compact singlephoton detector based on a CMOS SPAD pixel. The system is housed in a compact commercial 1-inch diameter aluminum tube, it has a user-friendly interface and it is fully programmable via a USB link from a remote computer. This solution can pave the way to new widespread applications where current bulky detectors are a practical limitation.

Experimental characterization proved the excellent performance of the instrument: DCR lower than $100 \mathrm{cps}$ for an active area diameter of $50 \mu \mathrm{m}, 55 \%$ detection efficiency at $420 \mathrm{~nm}$ and timing resolution better than $100 \mathrm{ps}$ (FWHM).

The design is scalable and can be integrated in portable and low-power systems that could spread the use of single-photon detectors in yet unexplored fields (point-of-care medical tools, life science, microscopy, etc.). Its state-of-the-art performance makes it suitable also for more established photon counting applications, such as fluorescence lifetime imaging (FLIM), Förster resonance energy transfer (FRET), fluorescence correlation spectroscopy (FCS), functional near-infrared spectroscopy (f-NIRS), brain imaging, optical mammography, molecular imaging, quantum information and many others, as a cost-effective alternative to present bulkier and more expensive commercial systems.

\section{REFERENCES}

[1] A. Yodh and B. Chance,"Spectroscopy and imaging with diffusing light," Phys. Today, vol. 48, no. 3, pp. 34-41, Mar. 1995.

[2] D. Contini, L. Zucchelli, L. Spinelli, M. Caffini, R. Re, A. Pifferi, R. Cubeddu, and A. Torricelli,"Review: brain and muscle near infrared spectroscopy/imaging techniques," J. Near Infrared Spectrosc., vol. 20, no. 2, pp. 15-27, Jan. 2012.

[3] W. Becker, A. Bergmann, M.A. Hink, K. König, K. Benndorf and C. Biskup, "Fluorescence lifetime imaging by time-correlated singlephoton counting," Microsc. Res. Technol., vol. 63, no. 1, pp. 58-66, Jan. 2004.

[4] I. Bargigia, A. Nevin, A. Farina, A. Pifferi, C. D’Andrea, M. Karlsson, P. Lundin, G. Somesfalean, and S. Svanberg, "Diffuse optical techniques applied to wood characterization," J. Near Infrared Spectrosc., vol. 21, no. 4, pp. 259-268, Sep. 2013.

[5] J. Johansson, S. Folestad, M. Josefson, A. Sparén, C. Abrahamsson, S. Andersson-Engels, and S. Svanberg, "Time-resolved NIR/Vis spectroscopy for analysis of solids: pharmaceutical tablets," Appl. Spectrosc., vol. 56, no. 6, pp. 725-731, 2002.

[6] D. Bronzi, F. Villa, S. Tisa, A. Tosi, F. Zappa, D. Durini, S. Weyers, and W. Brockherde, "100 000 Frames/s $64 \times 32$ Single-Photon Detector Array for 2-D Imaging and 3-D Ranging," IEEE J. Sel. Top. Quantum Electron., vol. 20, no. 6, pp. 1-10, Nov. 2014.

[7] R. H. Hadfield, "Single-photon detectors for optical quantum information applications," Nat. Photonics, vol. 3, pp. 696-705, Nov. 2009.

[8] D. Bronzi, S. Tisa, F. Villa, S. Bellisai, A. Tosi and F. Zappa, "Fast sensing and quenching of CMOS SPADs for minimal afterpulsing effects," IEEE Photon. Technol. Lett., vol. 25, no. 8, pp. 776-779, Apr. 2013.

[9] S. Tisa, F. Guerrieri, and F. Zappa, "Variable-load quenching circuit for single-photon avalanche diodes," Opt. Express, vol. 16, no. 3, pp. 22322244, Feb. 2008.

[10] W. Becker, "The bh TCSPC Handbook," Berlin, Germany: Becker \& Hickl GmbH, 5th Edition, 2012.

[11] "Micro Photon Devices PDM Datasheet." Internet: http://www.microphoton-devices.com/Products/Photon-Counters/PDM [Dec. 03, 2014].

[12] "ID Quantique ID100 Datasheet." Internet: http://www.idquantique.com/photon-counting/photon-countingmodules/id100-silicon-apd-single-photon-detector.html [Dec. 03, 2014].

[13] "Excelitas SPCM Datasheet." Internet: http://www.excelitas.com/Pages/Product/Single-Photon-CountingModules-SPCM.aspx [Dec. 03, 2014].

[14] F. Villa, D. Bronzi, Y. Zou, C. Scarcella, G. Boso, S. Tisa, A. Tosi, F. Zappa, D. Durini, S. Weyers, U. Paschen and W. Brockherde, "CMOS SPAD with up to $500 \mathrm{um}$ and $55 \%$ detection efficiency at $420 \mathrm{~nm}$," J. Mod. Optic, vol. 61, no. 2, pp. 102-115, Jan. 2014. 